\title{
SCHREIBSTUNDE AN DER TELEgRAFEnLINIE. ZUR GRENZE VON SCHRIFTLICHKEIT UND MÜNDLICHKEIT BEI CLAUde LÉVI-STRAuSS UND JACQUES DERRIDA

\author{
ALEXANDER HONOLD
}

In den Kultur- und Medienwissenschaften hat sich die Frage nach dem Verhältnis von Oralität und Schriftkultur als ein prosperierendes und kontrovers behandeltes Forschungsfeld erwiesen. Methodisch umstritten war und ist, ob hierbei die These einer genealogischen Vorrangstellung des mündlichen Kommunizierens den Ansatzpunkt bilden soll (so etwa die kulturanthropologische Position von Jack Goody und Walter Ong), oder ob Mündlichkeit als ein selbst schon mediengenerierter Effekt der Schriftkultur respektive ihrer Episteme - der »Grammatologie« - zu denken ist (so neben den einschlägigen Arbeiten Derridas auch Marshall McLuhan, Friedrich Kittler und Walter Mignolo).

Derridas Theorie der Schrift, die er in der Grammatologie ${ }^{1}$ entwirft, richtet sich gegen die These von der Dominanz und Vorgängigkeit des Mündlichen; sie ist insofern auch eine Theorie der Grenze zwischen Schrift und Mündlichkeit, die ihre Einsichten zu einem bemerkenswerten Teil auf das Material ethnographischer Autoren und seine kritische Lektüre gründet. Ich möchte deshalb zunächst die Einführung des Gegensatzes von Schriftlichkeit und Mündlichkeit im retrospektiven Reisebericht des französischen Ethnologen Claude Lévi-Strauss beleuchten, weil Derrida in seiner Lektüre der Traurigen Tropen ${ }^{2}$ diese doppelte Annäherung an die kommunikative und mediale Determiniertheit der ethnographischen Situation prägnant herausgestellt und zum Ausgangspunkt einer ideologiekritischen Analyse gemacht hat. Obwohl Derridas Hauptaugenmerk dabei dem Kapitel gilt, das mit »Leçon d'écriture« (also nicht

1 Jacques Derrida: De la grammatologie. Paris: Ed. de minuit 1967. Dt.: Grammatologie. Übersetzt von Hans-Jörg Rheinberger und Hanns Zischler. Frankfurt a.M.: Suhrkamp 1974.

2 Claude Lévi-Strauss: Tristes Tropiques. Paris: Plon 1955. Dt.: Traurige Tropen. Übersetzt von Eva Moldenhauer. Frankfurt a.M.: Suhrkamp 1978. 
»Schreibstunden«, wie in der deutschen Übersetzung) einschlägig betitelt ist, ${ }^{3}$ greift er in seiner Argumentation dezidiert auf die jener Schreibstunde korrespondierende Szene eines früheren Kapitels zurück, in der es um die Eigennamen der Nambikwara geht; die meisten Kritiker, die sich ausgehend von Derridas Lektüre mit Lévi-Strauss beschäftigt haben, sind ihm indes hierin gerade nicht gefolgt, somit auch nicht der plakativen Entgegensetzung und Symmetrie beider Szenen. Bevor auf das Argument Derridas näher einzugehen ist, soll deshalb zunächst die kompositorische und sachliche Korrespondenz der beiden anekdotischen Feld-Szenen selbst betrachtet werden.

Was Lévi-Strauss in seinem mehrmonatigen Forschungsaufenthalt bei den Nambikwara herausfindet, dieses ethnographische Wissen verdankt sich situativen Experimenten, über die in Form von dramaturgisch ausgestalteten Episoden berichtet, recht eigentlich also erzählt wird. Die Anekdote verbindet als pointierte erzählende Kurzform den subjektiven Arbeitsbericht über die Vorgehensweise des Feldforschers mit der motivischen Ausarbeitung eines bestimmten Themas bzw. ethnographischen Sachgehalts. Es geht in den beiden zentralen Anekdoten über den Aufenthalt bei den Nambikwara um die Auftrittsbedingungen von Medialität an der Nahtstelle zwischen Mündlichkeit und Schriftlichkeit. Die erste Episode thematisiert den Zugang zu den Eigennamen, die zweite den $\mathrm{Zu}-$ gang zur Schrift; die beiden Handlungssequenzen sind als pointierte, d.h. auf überraschende Effekte hin ausgerichtete Erzählungen dargeboten und verhalten sich in ihrem Aufbau wie spiegelsymmetrisch aufeinander bezogene Durchführungen eines medialen Leistungsvergleichs von Mündlichkeit und Schriftlichkeit.

Das Problem des Ethnographen ist, wie erwähnt, die besondere Schutzfunktion, die bei den Nambikwara auf dem Gebrauch der Eigennamen liegt. Im Namen akkumuliert sich ein arkanes Wissen über die mit ihm bezeichnete Person, das demjenigen, der über den Namen verfügt, eine hohe soziale Macht verleiht; deshalb fungiert der Eigenname als ein schützenswertes Gut, das nach außen hin, d.h. hier vor allem gegenüber dem ethnographischen Forscher, der strengen Geheimhaltung unterliegt.

3 Zur »Schreibstunde« selbst und dem medialen Aufeinandertreffen von Mündlichkeit und Schriftlichkeit in der kolonialen und ethnographischen Situation vgl. Michael Harbsmeier: Writing and the Other: Travellers' Literacy, or Towards an Archaeology of Orality. In: Karen Schousboe, Mogens Trolle Larsen (Hg.), Literacy and Society. Kopenhagen: Akademisk Forlag 1989, S. 197-228; Erhard Schüttpelz: Heischebräuche. Der >supplementäre symbolische Inhalt` der Schreibstunde von Claude Lévi-Strauss. In: Jürgen Fohrmann (Hg.), Rhetorik. Figuration und Performanz. Stuttgart, Weimar: Metzler 2004, S. 361-396. 
Was der Forscher statt dessen verwenden kann, sind die Aliasnamen, auf die sich die Indianer mit den Angestellten der Telegrafenlinie geeinigt hatten, stellvertretende Namen also, die ihrerseits aus einer Übergangszone hervorgehen und übersetzende Funktion haben. Aus dieser Behelfsmaßnahme geht deutlich hervor, daß der Name für beide Seiten eine strategische Bedeutung hat; dem arkanen Besitz des Namens steht der Ausschluß von diesem Arkanwissen gegenüber. Es gibt in dieser Begegnung keinen unvorbelasteten, keinen unpolitischen Namen, sondern stets schon den >nom de guerre stehenden Beobachter, zugleich aber auch die Zuordnung der Personen und ihrer Körper zur Ordnung der Zeichen. Als genuine Form der Stellvertreterschaft (die dann im Aliasnamen nochmals potenziert wird) hat der Eigenname Schrift-Funktion, obwohl er nur in mündlicher Präsenz vorhanden ist.

Der Ethnograph erzählt nun, wie es ihm mit Hilfe einer List gelang, sich in den geheimen Namenscode der Nambikwara systematisch >einzuloggen<.

Als ich eines Tages mit einer Gruppe von Kindern spielte, wurde eines der kleinen Mädchen von einer Gefährtin geschlagen; es flüchtete sich zu mir und flüsterte mir geheimnisvoll etwas ins $\mathrm{Ohr}$, das ich nicht verstand und mir mehrmals wiederholen ließ, so daß die Gegnerin das Treiben entdeckte und, offensichtlich wütend, herbeilief, um mir ihrerseits etwas zu verraten, das allem Anschein nach ein feierliches Geheimnis war. Nach einigen Bedenken und Fragen konnte es an der Bedeutung des Zwischenfalls keinen Zweifel mehr geben: das erste Mädchen hatte mir, um sich zu rächen, den Namen seiner Feindin verraten, und als diese es bemerkte, verriet sie mir nun ihrerseits den Namen des anderen Mädchens. Von nun an war es mir ein leichtes, die Kinder gegeneinander aufzubringen - ich gebe zu, auf etwas skrupellose Weise - und alle ihre Namen zu erfahren. Und nachdem zwischen uns einen Art Komplizenschaft entstanden war, erfuhr ich ohne große Mühe auch die Namen der Erwachsenen. ${ }^{4}$

Die Anekdote demonstriert, wie beschämend leicht die im Eigennamen akkumulierte Macht von seiten der Nambikwara auf den Ethnographen übergeht; sein Gegenüber sind hier kleine Mädchen: diejenigen Mitglieder der Gemeinschaft, die ohnehin auf der sozialen Skala ganz unten stehen. Sie befinden sich zudem durch interne Streitigkeiten in einer akuten Situation der Hilflosigkeit, die so dramatisch ist, daß sie es sogar auf sich nehmen, den Fremden als Schutzmacht anzurufen und auf ihre Seite zu ziehen. Das gewährte Vertrauen wird vom teilnehmenden Beobachter indes grob mißbraucht. Am schwächsten Glied ansetzend, gelingt es ihm,

4 C. Lévi-Strauss: Tristes Tropiques, S. 271f. 
nach und nach den Geheimcode der Eigennamen zu entschlüsseln. Die Vertraulichkeit wurde in dezidiert mündlicher Weise übermittelt, flüsternd sogar, am Ende aber stehen verwertbare Informationen, die der Forscher in sein Notizbuch eintragen kann. Erfolgreich hat er sich in ein internes Machtspiel der Indianer eingeschaltet; dies belegt, daß ihr System, das aus mündlicher Kommunikation bei gleichzeitiger mündlicher Geheimhaltung basiert, von der persönlichen Anfälligkeit bzw. Widerstandskraft jedes seiner Träger abhängig ist. Ganz anders nun das Medium Schrift, das in der zweiten, gegenläufig komponierten Anekdote seinen Auftritt hat.

Die »Schreibstunde« eröffnet mit dem heiklen Projekt einer Volkszählung. »Ich wollte nun«, schreibt Lévi-Strauss, »zumindest indirekt in Erfahrung bringen, wie groß die Nambikwara-Bevölkerung ungefähr war. $\aleph^{5}$ Hier wiederholt sich der Ehrgeiz einer Bestandsaufnahme, den der Ethnograph schon in bezug auf die Eigennamen an den Tag gelegt hatte, auf der Ebene einer statistisch-demographischen Erfassung der Gesamtpopulation. $\mathrm{Zu}$ dokumentieren ist auf diese Weise, so die Arbeitshypothese, ein vermutlich dramatischer Rückgang der Bevölkerung. Für die melancholische Rahmenerzählung vom Ende der Feldforschung, das zusammenfällt mit dem Ende der Beforschten, wäre das vermutete Resultat der Volkszählung eine wichtige positivistische Stützung. Andererseits setzt der inventarisierende Zugriff selbst eine nicht unproblematische Sozialtechnologie ein und trägt damit womöglich zusätzlich noch zur Schwächung der indianischen Lebenswelt bei; somit wäre die Frage nach der Zahl gleich ein doppelter Anlaß zur Melancholie.

Der Ethnograph wendet sich an den »Häuptling« und schlägt diesem vor, zur Durchführung einer solchen Bestandsaufnahme ein größeres Treffen »mit anderen, verwandten oder verbündeten Gruppen zu organisieren ${ }^{6}{ }^{6}$, um auf diese Weise einen gewissen Überblick gewinnen $\mathrm{zu}$ können. Sein Verhandlungspartner ist skeptisch, weil er gewaltsame Übergriffe befürchtet, die den Ruf der Nambikwara weiter in Mißkredit brächten, stimmt aber, von den in Aussicht gestellten Geschenken beeindruckt, letztlich zu und wird auf diese Weise zum Partner oder Komplizen einer hochgradig experimentellen Veranstaltung. Am Zielort angekommen, trifft die Exkursion auf ungefähr 75 dort versammelte Nambikwara, die Lage ist angespannt, die Stimmung gereizt. »Mehrere Eingeborene schienen noch nie einen Weißen gesehen zu haben, und ihr frostiger Empfang sowie die offenkundige Nervosität des Häuptlings ließen darauf schließen, daß er ihre Einwilligung wohl erzwungen hatte. ${ }^{7}$

5 Ebd., S. 288.

6 Ebd., S. 289.

7 Ebd., S. 290. 
Um die aufgeladene Begegnung zu deeskalieren, »drängt« LéviStrauss den Häuptling, mit der Verteilung bzw. dem wechselseitigen Austausch der mitgebrachten Geschenke zu beginnen. An dieser Stelle der Dramaturgie geht es nun offenkundig darum, wer Regie führt, wer die Definitionshoheit über das weitere Vorgehen innehat. Der Häuptling muß gleich an zwei Fronten dem drohenden Autoritätsverlust entgegenwirken, er muß die assoziierten Gruppen hinter sich scharen und gegenüber dem Ethnographen die eigene Souveränität hervorkehren. Beides zugleich betreibt er, indem er »ein Papier mit verschnörkelten Linien« hervorholt und nun fingiert, von diesem Dokument Anordnungen über die >korrekte<, das heißt dem allgemeinen Konsens entsprechende, mit den Stammesangehörigen vereinbarte und schriftlich niedergelegte Verteilung der Geschenke abzulesen: »dem einen, gegen Pfeil und Bogen, ein Buschmesser! dem anderen Perlen für seine Halsketten! ... Diese Komödie zog sich zwei Stunden hin. $\ll^{8}$

Zur Komödie wird das vom Häuptling ja nur kommissarisch inszenierte Spektakel des Gabentausches, weil er dabei nach einem Drehbuch vorzugehen behauptet, das gar nicht existiert. Weder sind die Modalitäten des Austauschs der Geschenke tatsächlich so verabredet worden, noch gibt es eine schriftliche Liste, nach deren Verfügungen vorgegangen werden könnte. Die dreiste und freche Anmaßung des Häuptlings, aus der Bedrängnis entstanden, in die ihn die Initiative des Ethnographen gebracht hatte, gipfelt nun allerdings in der vorgetäuschten Fähigkeit des Verfügens über die Schrift. Um die ganze Lächerlichkeit seiner Darbietung von vorneherein zu entblößen, hatte sich Lévi-Strauss bei der Einleitung dieser Anekdote beeilt vorauszuschicken, daß die Nambikwara und auch ihr Häuptling vollständig illiterat und sogar >agraphく sind. »Es läßt sich denken, daß die Nambikwara nicht schreiben können; aber sie zeichnen auch nicht, mit Ausnahme einiger punktierter oder Zickzacklinien auf ihren Kürbisbehältern. « ${ }^{9}$

Zum Ausschluß des Ethnographen durch die geheim und mündlich zirkulierenden Eigennamen erzählt dieser vergebliche Angriff des Indianerhäuptlings auf das weiße Schriftmonopol die Gegengeschichte. Schon vor dem öffentlichen Tauschritual hatte der Häuptling aus der Beobachtung des Beobachters den Schluß gezogen, die Überlegenheit des Forschers müsse unmittelbar mit seiner Schreibpraxis, mit dem Zusammenspiel von Notizbuch und Bleistift, zusammenhängen. Allerdings hatte der Ethnograph zuvor selbst die Indianer zur Nachahmung seines Schriftgebarens aufgefordert und, wenn das Wortspiel erlaubt ist, ")angestiftet«. Wie bei anderen Stämmen verteilt er »Papier und Bleistifte, mit denen

8 Ebd., S. 291.

9 Ebd., S. 290. 
zuerst niemand etwas anzufangen wußte; doch eines Tages sah ich sie alle damit beschäftigt, horizontale Wellenlinien auf das Papier zu zeichnen ${ }^{10}{ }^{10}$ Die graphische Technik, die den Ethnographen ausmacht, wird zum Gegenstand einer mimetischen Übernahme; der skurrile Effekt ist nichts anderes als das provozierte, daher auch prognostizierbare Echo einer entsprechenden Konditionierung: »sie schrieben, oder genauer: sie versuchten, ihren Bleistift in derselben Weise zu benutzen wie ich «. ${ }^{11}$

Anders als die listige Enteignung der Eigennamen ist die umgekehrte mimetische Annäherung nicht von Erfolg gekrönt; die schreibende Tätigkeit äußerlich nachzuahmen, bedeutet, von dem Geheimnis der Schrift desto nachdrücklicher ausgeschlossen zu sein. Mit der Korrektur bekräftigt Lévi-Strauss die Inkompetenz der Nambikwara und betont ihren nicht graduellen, sondern prinzipiellen Abstand zur Schrift. Die Schriftbenutzung selbst ist eine habitualisierte Grenzziehung; ganz egal, was der Ethnograph bei der Arbeit niederschreibt: allein daß er überhaupt schreibt, trennt ihn von den Beschriebenen. Die meisten der Indianer scheinen sich mit dem uninspirierten Nachahmen der schreibenden Hand zu begnügen; einzig dem Häuptling unterstellt der Ethnograph eine tiefergehende Einsicht in das mit der Schrift verbundene Geheimnis; die Schrift zu beherrschen, ist ein Herrschaftsattribut überhaupt und als solches gerade dem lokalen Machthaber verständlich. »Wahrscheinlich hatte er als einziger die Funktion der Schrift begriffen. So hat er mich um einen Notizblock gebeten, und wenn wir nun zusammen arbeiten, sind wir gleichartig ausgerüstet. ${ }^{12}$

Der Unterricht in der Schreibstunde ist, darüber muß sich auch der Kulturbringer selbst klar sein, von vornherein nur ein schlechter (weil despektierlicher) Witz; und der Häuptling beantwortet ihn als einziger auf die adäquate Weise, indem er den Witz zurückgibt und sich als erfolgreichen Schüler präsentiert. Dann aber muß der Lehrer die Fiktion des gemeinsamen Schriftverkehrs ebenfalls anerkennen und bekräftigt somit wider besseres Wissen ausdrücklich den eigenen Lehrerfolg, den ihm der Häuptling immer nur halbherzig vorzuspielen vermochte.

Halb fällt er selbst auf seine Komödie herein; jedesmal, wenn seine Hand eine Linie zu Ende zieht, prüft er sie ängstlich, als müsse ihre Bedeutung sofort daraus hervorspringen, und auf seinem Gesicht malt sich immer wieder die gleiche Enttäuschung. Aber das will er nicht wahrhaben, und zwischen uns besteht eine stille Übereinkunft, daß seine Kritzeleien einen Sinn haben, den zu entziffern

10 Ebd., S. 290.

11 Ebd., S. 290.

12 Ebd., S. 290f. 
ich vortäusche; der mündliche Kommentar folgt immer so prompt, daß ich nicht um nähere Erläuterungen zu bitten brauche. ${ }^{13}$

Dieser ironischen Szene läßt Lévi-Strauss dann jene folgen, in welcher der Häuptling seinen >Schrifterwerb< öffentlich demonstriert, nun sein neues Komödiantentum zum ersten Mal auch gegenüber den Mitgliedern des eigenen Stammes erprobend. Doch auf die »Mystifizierung« reagieren die Zuschauer ziemlich ungehalten, und fast wäre die ganze Situation vollständig entgleist.

Dem Abenteuer läßt Lévi-Strauss nun eine Interpretation folgen, die der halb tragischen, halb lächerlichen Figur des von beiden Seiten desavouierten Häuptlings im nachhinein recht gibt. Denn hatte der gelehrige Schüler nicht sogar besonders gut verstanden, welche symbolische Aufladung dem Schriftgebrauch zukommt? Unabgelenkt von primärem Sinnverstehen hatte der Häuptling nicht die Kommunikationsform affirmiert, sondern nur die gestische Anmutung und soziale Wirkung des Mediums beobachtet - er hatte sich also im methodischen Sinne ethnographisch (und obendrein im Sinne des strukturalistischen Paradigmas) ${ }^{14}$ verhalten. »Ein Eingeborener, der noch dem Steinzeitalter anzugehören schien, hatte erraten, daß das große Verständigungsmittel, auch wenn er es nicht verstand, anderen Zwecken dienen konnte. $\ll^{15}$ Der Außenstehende wird zum Kronzeugen eines von Lévi-Strauss geführten Argumentes der Schriftkritik. Ziel dieser Kritik ist die ethnologische Gepflogenheit, eine scharfe Differenz zwischen Kultur und Barbarei mit Hilfe des Kriteriums der Schriftkenntnis zu ziehen. Nur schreibende Völker, so die auf dieser Unterscheidung fußende Hierarchisierung, seien in der Lage, »alte Erwerbungen zu kumulieren«, während »die schriftlosen Völker Gefangene einer schwankenden Geschichte bleiben «. ${ }^{16}$ Dieser Aufteilung widerspricht Lévi-Strauss vehement; im Hintergrund steht unverkennbar die geschichtsphilosophische Position Jean-Jacques Rousseaus. Setzt man als historische Basis aller Entwicklung die neolithische Revolution und ihre Errungenschaft der Agrikultur an, so lautet das Gegenargument,

13 Ebd., S. 291.

14 Erhard Schüttpelz liest die Reaktion des Häuptlings in dieser Szene als eine »Erkenntnis, die das strukturalistische Credo praktiziert«, indem sie die symbolische Funktion des Mediums `kongenial`, nämlich wiederum symbolisch, durchschaut. »Es ist daher nur konsequent, daß ein Kommunikationsmittel, dessen erste Funktion nicht das Verstehen, sondern die Vermehrung von Prestige ist, vom Häuptling eben nicht verstanden, sondern >erraten oder >diviniert` wird « (Schüttpelz: Heischebräuche, S. 368).

15 C. Lévi-Strauss: Tristes Tropiques, S. 293.

16 Ebd., S. 293. 
dann ist damit die Schrift als kultureller Gründungsfaktor außer Kurs gesetzt, da sie erst nach dieser Entwicklungsstufe auftritt. Der zivilisatorische Fortschritt ist keineswegs kongruent mit dem Schriftgebrauch, wohl aber letzterer mit der Installierung von Klassengesellschaften und Ausbeutung.

Wenn man das Auftauchen der Schrift mit gewissen Merkmalen der Kultur in Beziehung bringen will, muß man in einer anderen Richtung suchen. Das einzige Phänomen, das sie immer begleitet hat, ist die Gründung von Städten und Reichen, das heißt die Integration einer großen Zahl von Individuen in ein politisches System sowie ihre Hierarchisierung in Kasten und Klassen. [...] Wenn meine Hypothese stimmt, müssen wir annehmen, daß die primäre Funktion der schriftlichen Kommunikation darin besteht, die Versklavung zu erleichtern. ${ }^{17}$

Die weitreichenden Spekulationen über den Zusammenhang von Schriftund Machttechnik, Klassenspaltung und Unterwerfung sind in dieser historischen Allgemeinheit weder beweisbar noch widerlegbar. Sie sind, wie Derrida betont, Teil eines traditionsreichen philosophischen Projektes, das die Rehabilitierung einer als ursprüngliche und authentische Ausdrucksform postulierten Mündlichkeit betreibt; Oralität gilt in dieser Tradition als genealogisch vorgängiges Sprachsystem, das weder als schriftgeneriert noch als schriftanalog zu denken sei. Schrift, so jedenfalls gibt die Schreibstunde zu verstehen, ist die Signatur des Eindringlings und des Kolonisators in ein bereits existierendes, intaktes kommunikatives System.

Die pauschale, wenngleich selbstkritisch intendierte Identifikation des Herrschaftsinstrumentes der Schrift mit dem kolonialen und wissenschaftlichen Zugriff der Europäer auf andere Kulturen (»the pen itself is a kind of sword «, ${ }^{18}$ faßt Michael Harbsmeier diese Lehre der »Leçon« zusammen) ist aus ethnographiegeschichtlicher Sicht nicht unwidersprochen geblieben. Der erste Einwand gegen Lévi-Strauss lautet, daß seine Analyse der medientechnischen Überlegenheit erst eine relativ späte Entwicklung im kolonialen Kulturkontakt beschreibt und kein Apriori des first contact darstellt, sondern auf eine veränderte Einstellung innerhalb des kolonialen Blicks zurückgeht. Wie Harbsmeier an ethnographischen Zeugnissen von Reisenden seit Beginn der amerikanischen Kolonialisierung ausführt, spielt in den frühen Dokumenten die mediale Seite der kommunikativen Praxis bei der Wahrnehmung der fremden Kultur keine manifeste Rolle. Der Unterschied zwischen Mündlichkeit und Schriftlichkeit wird als ethnisches Differenzkriterium erst im 18. Jahr-

17 Ebd., S. 294.

18 Harbsmeier: Writing and the Other, S. 199. 
hundert im ethnographischen Diskurs etabliert (parallel zur terminologischen Umstellung von >wild ‘ auf >primitiv $<$ ), ein Reflex auch der zunehmend schriftfixierten Arbeitsweise und Dokumentationspraxis auf seiten der (ihre medientechnische Überlegenheit somit ausstellenden) Reisenden. ${ }^{19}$ Der zweite, hierauf fußende Einwand ist literarisch-topologischer Art. Er erkennt in Lévi-Strauss' Schriftkritik die »Travestie und Demontage « einer in vielen Reiseberichten kolportierten Wanderanekdote, die in wechselnder Einkleidung jeweils die Überlegenheit des Schriftgebrauchs vorführt, indem sie kleine Unterschlagungen eines für Transportdienste eingesetzten >Indianers` durch das ihn begleitende Schreiben am Ziel der Reise auffliegen läßt. Obwohl niemand unterwegs den begangenen Mundraub sehen konnte, weiß der weiße Mann am Ende des Transportweges aufgrund des $>$ Lieferscheins $\$ genau, ob etwas fehlt, und wie viel. Das Wunder des Regiments über Abwesenheit und Distanz hinweg $^{20}$ hat für Schriftkundige nichts Mysteriöses, für die hiervon Ausgeschlossenen ist es ein Zeichen der unerklärlichen fremden Machtfülle. Der eingeführten Anekdote nun habe Lévi-Strauss zwar mit dem demonstrativen Schrifteinsatz des Ethnologen eine weitere Evidenz hinzugefügt; mit der mißlingenden und von der eigenen Gruppe mißbilligten Imitation des Schreibakts durch den Häuptling aber habe Lévi-Strauss die Machtbalance der Szene gleichsam umgedreht und den Geltungsbereich der Schriftkompetenz in einer tropischen Karnevalisierung untergraben bzw. an seine Grenzen geführt. ${ }^{21}$ Die Kritik der Schriftkritik wendet sich an diesem Punkt von der praxeologischen Seite des ethnographischen Kontakts zur performativen Qualität des Medieneinsatzes und der Frage, inwieweit darin aus systematischer Sicht eine aporetische Dimension des Gegensatzes von Oralität und Literalität zum Ausdruck kommt.

19 Harbsmeier: Writing and the Other, S. 219.

20 Das Problem der über globale Distanzen sich erstreckenden Kontrolle durch Schrift und Information als einer Rahmenbedingung des entwickelten Kolonialismus diskutiert Thomas Richards: The Imperial Archive. Knowledge and the Fantasy of Empire. London, New York: Verso 1993. Zur Bedeutung der Schrift im kulturellen Prozeß der Kolonisierung Lateinamerikas vgl. Roberto Gonzáles Echevarria: Myth and Archive. A Theory of Latin American Narrative. Cambridge: Cambridge University Press 1990, S. 43-92.

21 »Offensichtlich ist die von Lévi-Strauss erzählte Schreibstunde [...] eine Kontrafaktur der Wanderanekdote von der schriftlichen Überlegenheit europäischer Reisender und Kolonisten«, inszeniert und erzählt mit dem Ziel, »den ungleichen Tausch der Schrift auszugleichen« (Schüttpelz: Heischebräuche, S. 373). 
Damit zurück zur szenischen Dramaturgie des Schreibunterrichts bei den Nambikwara. Die fragile und peinliche Komödie des Häuptlings demaskierte ein dem Schriftgebrauch inhärentes Unterwerfungspotential. Deshalb mußte die zweite, vom Häuptling betriebene Leçon d'écriture, die >Lektüre der Schrift`also, schiefgehen, indem sie das in der ersten, durch den Ethnographen erteilten Schreiblektion nur implizit mitschwingende Gewaltverhältnis dann offen und öffentlich ausagierte. Im mehrdeutigen Titel der »Leçon d'écriture« bilden Unterweisung und Lektüre die beiden Extrem-Amplituden; dazwischen findet sich Platz für allerlei Lesarten, Lesungen, Vorlesungen, Warnungen und didaktische Erkenntniseffekte. $^{22}$ Am Ende hat jeder jedem eine Lektion erteilt. Der Ethnologe dem Häuptling über die Macht der Schrift, aber auch über die Ohnmacht des Schreibenden jenseits der Schrift; der Häuptling den Nambikwara über die Grenzen der Mimesis und die Unverfügbarkeit des Sinns; die Indianer dem Häuptling und dem Ethnographen über die Unverfügbarkeit ihres Willens zu Tauschgeschäften; die Schrift dem Häuptling über die Möglichkeiten des Fehllesens, des fehlenden Lesens und der verpaßten Lektionen; und schließlich Häuptling, Indianer und Schriftmedium zusammen dem Ethno-Graphen über sein eigenes Tun und die darin zum Ausdruck kommende, problematische Privilegierung einer die Oralität bekämpfenden (und zugleich bewundernden) Schriftkultur.

Für die melancholische Bilanz der Traurigen Tropen nimmt die in dieser Episode am prägnantesten ausgeführte Entzauberung ethnographischer Souveränität eine Schlüsselstellung ein, denn hier richtet sich die Kritik auf beide tragenden Elemente zugleich, auf den Ethnozentrismus und auf die graphische Kompetenz. Mithilfe des unbestechlichen fremden Probanden negiert die Versuchsanordnung ein zentrales Ideologem der Schrift, nämlich die Suggestion der spontanen Sinnemergenz. Des Häuptlings Enttäuschung, als aus den Wellenlinien eben keine Bedeutung sofort hervorspringt, drückt nicht (nur) den lächerlichen Irrtum eines hilflosen Autodidakten aus, sondern eine semiotisch berechtigte Skepsis. Denn würde die Schrift, wie sie sich anheischig macht, über Oralität als ein Sekundärphänomen souverän verfügen können, dann bliebe sie bei der Lektüre ja wohl nicht stumm, wie es zum Verdruß des Häuptlings der Fall ist.

Das Erbe des Phonozentrismus im hermeneutischen Selbstverständnis der Geisteswissenschaften ist hartnäckig: Wenn wir Texte verstehen wollen, dann müssen wir sie >zum Sprechen bringen`; was wir über sie sagen, bemißt sich in seinem Wahrheitsgehalt an einer ausschließlich

$22 \mathrm{Zu}$ den changierenden Bedeutungen der Leçon d'écriture vgl. auch Schüttpelz: Heischebräuche, S. 362f. 
akustischen Performanz, es muß »stimmen $«{ }^{23}$ In der »Leçon d'écriture« hat dem Schreiben sein letztes Stündchen geschlagen, sie hat, wie die Traurigen Tropen im ganzen, Endspiel-Charakter. Das schriftliche Dokument kann die direkte und unmittelbare Redeform nicht ersetzen, es muß, um Sinn generieren zu können, immer durch mündliche Aktivitäten supplementiert werden. In die enge Kopplung von Stimme und Sinn, so lernt und beweist der Häuptling, kann die Schrift als Medium gar nicht dazwischentreten. Insofern handelt die Schreibstunde mit dem Mißerfolg des Häuptlings und dem anschließenden Verlorengehen des Ethnographen von nichts als Umwegen und Abwegen. Die Schrift ist überflüssig und kraftlos, zugleich aber wird sie als gewaltsam und kolonial inkriminiert.

Die beiden Negativaspekte der Schrift - hohler Bluff und Gewaltverhältnis - scheinen sich zu widersprechen. Beides geht nur dann zusammen, wenn die Schrift auf ein bestimmtes medientechnisches Paradigma reduziert wird, und an diesem Punkt kommt nun Derridas dekonstruktive Lektüre der Traurigen Tropen zum Zuge. Die Ernte der Dekonstruktion beginnt damit, im untersuchten Text Zweifel zu sähen. Sind die Nambikwara wirklich eine schriftlose Kultur? Und: Gelingt es dem Ethnographen tatsächlich, sich vom gewaltsamen Moment des Schriftgebrauchs zu distanzieren? Derrida wirft die Frage auf, »inwieweit es legitim ist, jene >Stricheleien « und >Zickzacklinien` auf den Kürbissen, die mit so wenigen Worten in den Traurigen Tropen in Erinnerung gerufen werden, nicht Schrift zu nennen. $\ll^{24}$ Als graphematische Strukturen haben sie wohl doch einen vergleichbaren Status wie andere voralphabetische Zeichenformen und sprächen im Gegenteil für eine Erweiterung des Schriftverständnisses, das dann auch dingsymbolische Codes einbezieht und sowohl die »Artikulation graphischer Formen« zu seinen Gegenständen zählt wie auch die Untersuchung »graphischer Substanzen (Holz, Wachs, Leder, Stein, Tinte, Metall, Pflanzenstoffe) oder Instrumente (Stift, Pinsel usw.) ${ }^{25}$

An solchen Stellen ist spürbar, daß Derridas Konzept der Grammatologie selbst einem genuin ethnologischen Kontext entnommen ist, der

23 Existentielle Gewißheiten liegen auch heute noch nur in seltsamer Oralitätsfixierung vor, so etwa das televisionäre Ritual der allwöchentlichen Ziehung der Lottozahlen, bei denen Sein und Sinn erst im Akt der performativen Verkündung zusammenfallen; denn bei ihrer öffentlichen Kundgabe ist es, der Bildlastigkeit des Mediums zum Trotze, offenbar rhetorisch unabdingbar, daß die Lottozahlen in der dann bekanntgegebenen Weise, so wörtlich, »lauten«.

24 J. Derrida: De la grammatologie, S. 193.

25 Ebd., S. 157. 
durch die Schnittstelle von Oralität und Literalität markiert ist. ${ }^{26}$ Dieser alle graphischen Kulturtechniken umfassende Schriftbegriff hat bei Derrida selbst einen utopischen Index. Es geht darum, »endlich das zu lesen, was in den vorhandenen Bänden schon immer zwischen den Zeilen geschrieben stand «. ${ }^{27}$ (155) Es geht um die Befreiung der graphischen Bildlichkeit in ihrer sinnlichen Evidenz von der abstrakten Herrschaft der Linie. Das Programm der Grammatologie ist an diesem Punkt eminent politisch und progressistisch. Es verbündet sich mit dem Ende eines bestimmten, restriktiven Schriftkonzepts; es setzt seine Hoffnung auf revolutionäre »Erschütterungen «, die »das lineare Modell - unter dem wir das epische Modell verstehen - nach und nach zerstören «. ${ }^{28}$ Dreißig Jahre vor dem Zeitalter der Hypertexte formuliert Derrida: »Was es heute $\mathrm{zu}$ denken gilt, kann in Form der Zeile oder des Buches nicht niedergeschrieben werden. $\ll^{29}$

Im hier diskutierten Zusammenhang ist allerdings nicht die Zukunftsvision der Grammatologie von primärem Interesse, sondern die historische Rekonstruktion dessen, was Derrida als Dominanz des linearen oder epischen Modells attackiert. Und abermals ist diese Rekonstruktion nicht ohne jenen Bezug zur Ethnologie und Anthropologie herstellbar, auf dem auch Derridas Einsichten fußen. Mit Verweis auf die Forschungen des Anthropologen Leroi-Gourhan bewegt sich Derrida in eine geschichtliche Zone zurück, in der Ideographie und Piktographie noch nicht voneinander geschiedene graphische Praxisformen darstellten; in ihrem komplexen Zeichenverbund sieht Derrida eine »Einheit« verwirklicht, »welche durch die lineare Schrift aufgebrochen wurde. Um aber den Zugang zu dieser Einheit, zu dieser ganz andersartigen Einheitsstruktur wiederaufzufinden, müssen [wie er mit Leroi-Gourhan fordert] >viertausend Jahre linearer Schrift، Schicht für Schicht abgetragen werden. « Wie Lévi-Strauss eine Geschichte vom Verschwinden der Indianervölker Brasiliens und vom Ende der Feldforschung erzählt, so skizziert Derrida in solchen Bemerkungen eine Entstellungsgeschichte der Schrift. Die »Vergangenheit einer nicht-linearen Schrift $\aleph^{30}$ muß als Kontrastfolie erst wieder präsent gemacht werden, will man verstehen, wie die abendländische Reduktion auf Linie, Buch und Zeile das graphische Paradigma transformiert, eingeengt und dynamisiert hat. In der Technik des Schreibens

26 Derrida verweist auf I.J. Gelb: A Study of Writing. The foundation of Grammatology, London: Routledge and Kegan Paul 1952, der den Begriff im anthrolologischen Kontext einführt (ebd., S. 13).

27 J. Derrida: De la grammatologie, S. 155.

28 Ebd.

29 Ebd.

30 J. Derrida: De la grammatologie, S. 151. 
hielt ein lineares Ablaufschema Einzug, das sowohl konkrete Phänomene betrifft, etwa in der Motorik der Hand, aber auch abstrakte Vorstellungen des menschlichen Seins und der Geschichte. Aus Heideggers Metaphysik-Kritik bringt Derrida hierfür den Aspekt der linearen, geradlinigen Zeitlichkeit in Anschlag, der »die ganze Ontologie von Aristoteles bis Hegel im Innersten $~^{31}$ determiniere. $»$ Linearisierung $\aleph^{32}$ der Schrift, das bedeutet die Temporalisierung, Vektorisierung und Teleologisierung dieses Mediums; sie >verkürzt` gleichsam die Partitur eines komplexen graphischen Ganzen auf die strikte, von links nach rechts führende Zeilenanweisung des einen zurückzulegenden Weges.

Die Zeile als Zurichtungsform der Schrift ist ein zwiefach codierter Zeitpfeil; sie ist einerseits Spur im Hinblick auf den vorausgegangenen Schreibakt, andererseits Bahnung für den einsinnigen Lektüreweg, der dieser Spur zu folgen hat. Eine beziehungsreiche Isomorphie zwischen linearer Schrift und temporalisiertem Weg: das ist es, was Derrida im Begriff des »epischen« Modells anklingen läßt. Die Narration als elementare literarische Technik ist eine auf Basis geometrischer Linearität ausfabulierte Zeitlichkeit, eine graphische Version der bzw. von Geschichte, die im Zurücklegen einer Wegstrecke greifbar und faßlich wird. Der Protagonist eines Erzählwerkes, wie es auch die Traurigen Tropen darstellen, bewegt sich durch die Zeilen des geschriebenen Textes und des gedruckten Buches auf sein Ziel und sein Ende hin, und er tut dies, wenn wir das große Exkursionsprojekt des Forschungsreisenden beim Wort nehmen, genau auf der Spur einer kolonialen Erschließungstrasse, nämlich der Telegrafenlinie. »Eindringen ${ }^{33}$ ist, neben dem Schreiben, die wichtigste Beschäftigung des Ethnographen, so hebt Derrida hervor; eindringen in ein Gebiet, das ohnehin schon von einer Trasse durchzogen ist, deren Spur es also zu verfolgen, mit anderen Worten: zu lesen und nachzuschreiben gilt. »[...] die Möglichkeit des Weges und der Differenz als Schrift müßten einmal aufeinander reflektiert werden ${ }^{34}{ }^{34}$, denkt Derridas Text so vor sich hin. An diesem Punkt schießen die intrinsische Leitästhetik der brasilianischen Telegrafenlinie und Derridas Linearitätskonzept zum Entwurf einer neuen Lektüre der Traurigen Tropen zusammen. Dabei würden aufeinandertreffen: »Die Geschichte der Schrift und die Geschichte des Weges, des Durchbruchs, der via rupta, des durchbrochenen, gebahnten Weges, [...] des von der Öffnung gezeichneten Raums

31 Ebd., S. 153.

32 »Der Begriff der Linearisierung ist weitaus wirksamer, genauer und inhärenter als alle anderen, welche man gewöhnlich für die Klassifikation der Schriften und zur Beschreibung ihrer Strukturen heranzieht« (ebd., S. 152).

33 Ebd., S. 188.

34 Ebd., S. 188. 
[...], des Abweichens von der Natur und der raumgreifenden Gewalt [...] im natürlichen wilden Wald. « ${ }^{35}$ Der überbordende Wildwuchs des eigenen Metapherndickichts hat Derridas flüchtigen, faszinierenden Gedanken fast schon wieder verschlungen, ehe ihm der Satz einen Weg bahnen konnte. Für ausgemacht aber hält Derrida, es lasse sich kaum vorstellen, »daß der mögliche Zugang zur straßenartigen Trasse nicht gleichzeitig auch ein Zugang zur Schrift ist $\aleph^{36}$. Die Pfade, die im wilden Urwald fehlgehen, führen unweigerlich auf die Spur eines epischen Trajektes, das Feldforschung in Erzähltext verwandelt. Was die Traurigen Tropen als Textphänomen evozieren, ist die erneute und mit jeder Lektüre wiederholte Durcharbeitung jener Urszene des Ethnographen, die in der Lektion über die Fremdheit der Schrift besteht. Mit nochmals anderen Worten: Wie man in den Wald hineinrief, so schreibt er zurück.

35 Ebd., S. $188 \mathrm{f}$.

36 Ebd., S. 189. 